\title{
Liver Manipulation Causes Hepatocyte Injury and Precedes Systemic Inflammation in Patients Undergoing Liver Resection
}

\author{
Marcel C. G. van de Poll · Joep P. M. Derikx · Wim A. Buurman · \\ Wilbert H. M. Peters · Hennie M. J. Roelofs · Stephen J. Wigmore • \\ Cornelis HC Dejong
}

Published online: 1 August 2007

(C) Société Internationale de Chirurgie 2007

\begin{abstract}
Background Liver failure following liver surgery is caused by an insufficient functioning remnant cell mass. This can be due to insufficient liver volume and can be aggravated by additional cell death during or after surgery. The aim of this study was to elucidate the causes of hepatocellular injury in patients undergoing liver resection. Methods Markers of hepatocyte injury (AST, GST $\alpha$, and L-FABP) and inflammation (IL-6) were measured in plasma of patients undergoing liver resection with and without intermittent inflow occlusion. To study the separate involvement of the intestines and the liver in systemic LFABP release, arteriovenous concentration differences for L-FABP were measured.

Results During liver manipulation, liver injury markers increased significantly. Arterial plasma levels and
\end{abstract}

Marcel C. G. van de Poll, Joep P. M. Derikx contributed equally to this work.

M. C. G. van de Poll - J. P. M. Derikx .

W. A. Buurman · C. H. Dejong

Department of Surgery, University Hospital Maastricht and

Nutrition and Toxicology Research Institute (NUTRIM),

Maastricht University, Maastricht, The Netherlands

W. H. M. Peters - H. M. J. Roelofs

Department of Gastroenterology, University Medical Center

Nijmegen, Nijmegen, The Netherlands

S. J. Wigmore

Liver Unit, Queen Elisabeth Hospital, Birmingham, UK

M. C. G. van de Poll ( $₫)$

Department of Surgery, Maastricht University, P.O. Box 5800,

6202 AZMaastricht, The Netherlands

e-mail: MCG.vandepoll@ah.unimaas.nl transhepatic and transintestinal concentration gradients of L-FABP indicated that this increase was exclusively due to hepatic and not due to intestinal release. Intermittent hepatic inflow occlusion, anesthesia, and liver transection did not further enhance arterial L-FABP and GST $\alpha$ levels. Hepatocyte injury was followed by an inflammatory response.

Conclusions This study shows that liver manipulation is a leading cause of hepatocyte injury during liver surgery. A potential causal relation between liver manipulation and systemic inflammation remains to be established; but since the inflammatory response is apparently initiated early during major abdominal surgery, interventions aimed at reducing postoperative inflammation and related complications should be started early during surgery or beforehand.

Liver failure is a severe complication of liver surgery, occurring when there is insufficient remnant cell mass postoperatively. This can be due to excessive resection, leaving a too small remnant liver volume, but in some cases liver failure occurs in patients with a seemingly sufficient remnant liver volume [1]. In these cases the functional capacity of the remaining cell mass is probably impaired by secondary processes. Recognition and modulation of factors impairing cell survival is crucial to enhance liver function following liver surgery. In this context much attention has been paid to the effects of ischemia and reperfusion.

Ischemia-reperfusion is a frequently encountered phenomenon in liver resection, caused by temporary occlusion of blood flow to the liver (Pringle maneuver), which is a popular way to reduce blood loss during parenchymal liver transection. Ischemia-reperfusion induces energy depletion and generation of reactive oxygen species. Al- 
Table 1 Patient characteristics

\begin{tabular}{lll}
\hline & $\begin{array}{l}\text { Hepatectomy } \\
\text { w/o IPM }(n=11)\end{array}$ & $\begin{array}{l}\text { Hepatectomy } \\
\text { with IPM }(n=9)\end{array}$ \\
\hline Age $(\mathrm{yr})$ & $57(36-72)$ & $54(33-75)$ \\
Male:Female & $9: 2$ & $5: 4$ \\
AST (IU/L) & $26(13-40)$ & $23(7-37)$ \\
LDH (IU/L) & $337(1-558)$ & $377(295-490)$ \\
Albumin $(\mathrm{g} / \mathrm{L})$ & $39.0(33.9-43.9)$ & $37.6(33.7-43.9)$ \\
CRP $(\mathrm{mg} / \mathrm{L})$ & $3.5(<1.0-8.7)$ & $5.0(<1.0-8.7)$ \\
Bilirubin $(\mu \mathrm{mol} / \mathrm{L})$ & $13.4(7.7-18.7)$ & $12.9(8.4-21)$ \\
\hline
\end{tabular}

IPM = intermittent Pringle maneuver

Blood chemistry was obtained during routine assessment one day preoperatively. Values are expressed as median (range).

though several endogenous protection mechanisms aimed at supporting cell survival are activated during ischemiareperfusion [2], excessive energy depletion or oxidative stress ultimately results in apoptotic or necrotic cell death.

Interestingly, it has been shown that plasma levels of markers for hepatocellular injury increase before liver transection and before application of the Pringle maneuver, suggesting that factors other than ischemia-reperfusion may cause hepatocyte demise during liver surgery [3]. In this context a role has been proposed for the hepatotoxic effects of anesthesia [4], the effects of systemic inflammation, probably secondary to intestinal manipulation [5], and the effects of manipulation of the liver itself during perihepatic dissection and mobilization [6]. The aim of this study was to elucidate the causes of early hepatocellular injury in patients undergoing liver resection.

\section{Methods}

\section{Patients}

Patients undergoing liver resection for secondary tumors in an otherwise healthy liver were studied (Table 1). All patients were anesthetized according to institutional routines using isoflurane and propofol. Surgery was commenced using a subcostal bilateral incision. Olivier retractors (Copharm, Abcoude, Holland) or Omni-Tracts were used to improve exposure. Before parenchymal division the liver was mobilized as described elsewhere, followed by intraoperative ultrasound [7]. Cholecystectomy was performed routinely before liver transection. All patients had indwelling radial artery catheters placed for continuous monitoring of arterial blood pressure and blood sampling. Patients were nonrandomly assigned to one of two protocols (described below). Assignment was based upon the surgeon's preference of using the Pringle maneuver or not. If applied, an intermittent Pringle maneuver (15 $\mathrm{min}$ of ischemia, $5 \mathrm{~min}$ of reperfusion) was used by rubberband ligation of the hepatoduodenal ligament.

Effects of intermittent Pringle maneuver on hepatocellular injury

From nine patients undergoing liver resection with intermittent Pringle maneuver, arterial blood was sampled preoperatively before and after each period of $15 \mathrm{~min}$ of ischemia and $5 \mathrm{~min}$ of reperfusion and 90 minutes postoperatively. Systemic concentrations of two markers of hepatocellular injury, aspartate amino transferase (AST) and glutathione-s-transferase- $\alpha$ (GST $\alpha$ ), were measured as well as plasma levels of liver-type fatty acid binding protein (L-FABP). Methods of blood processing and laboratory analyses are described below. In addition, plasma levels of the inflammatory cytokine interleukin-6 (IL-6) were measured as described below.

\section{Source and fate of L-FABP}

From ten patients undergoing liver resection without the Pringle maneuver, arterial blood was sampled preoperatively and before liver transection. Simultaneous with the second arterial blood sample, blood was drawn from the portal vein, a hepatic vein, and the right renal vein by direct puncture to assess concentration gradients across organs. It should be noted that at this timepoint surgical procedures were identical in all patients, irrespective of the eventual application of a Pringle maneuver. L-FABP plasma concentrations were measured as described below and arteriovenous concentration differences were calculated to study the contribution of the intestines and the liver to systemic L-FABP release and to study renal clearance of L-FABP. Renal clearance was calculated by dividing the arteriovenous concentration gradient by the arterial concentration (uptake/influx). This quotient was multiplied by the percentage of blood flowing through the kidney to calculate fractional plasma clearance.

Effects of anesthesia and intestinal manipulation

To differentiate the effects of anesthesia induction, laparotomy, and intestinal manipulation from the effects of liver manipulation, a control group consisting of four patients undergoing lower abdominal surgery (1 rectopexia, 2 proctectomy, 1 sigmoidal resection) was studied. Plasma was sampled before surgery and at 40-min intervals during surgery to determine L-FABP concentrations. 
Blood processing and analysis

Blood samples were collected in prechilled EDTA vacuum tubes (BD vacutainer, Becton Dickinson Diagnostics, Aalst, Belgium) and kept on ice. Blood was centrifuged at $4000 \mathrm{~g}$ for $10 \mathrm{~min}$. Plasma was immediately stored at $-80^{\circ} \mathrm{C}$ until analysis. L-FABP and IL-6 were determined using commercially available enzyme-linked immunosorbent assays (ELISA) (kindly provided by Hycult Biotechnology, Uden, The Netherlands), GST $\alpha$ was measured by ELISA as described earlier $[8,9]$. AST was determined by the clinical chemistry laboratory of the University Hospital Maastricht.

Ethics

The studies were approved by the Medical Ethics Committee of the University Hospital Maastricht and all subjects gave written informed consent.

\section{Statistics}

Normality of all data obtained was verified by Lillieford's test (all $p>0.10)$. Data are presented as mean with standard error of the mean (SEM). A paired $t$ test was used to test the significance of changes in the various plasma concentrations. Arteriovenous concentration gradients were tested versus a theoretical mean of zero using a one-sample $t$ test [10]. Pearson's test for correlations was used to test the significance of correlations. Statistical calculations were made using Prism 4.0 for Windows (GraphPad Software Inc., San Diego, CA). A $p$ value less than 0.05 was considered to indicate statistical significance.

\section{Results}

\section{Patient outcome}

No signs of organ failure or other major complications were observed postoperatively during the hospital stay of all patients.

Estimation of hepatocellular injury and intestinal injury after organ manipulation

\section{Systemic levels of damage markers}

During assessment of resectability, prior to organ transection, mean arterial L-FABP plasma concentration increased 55-fold ( $p=0.002$ ) (Fig. 1a). Surprisingly, arterial L-FABP levels did not change significantly during subsequent intermittent Pringle maneuver, indicating that neither intermittent ischemia-reperfusion nor liver transection
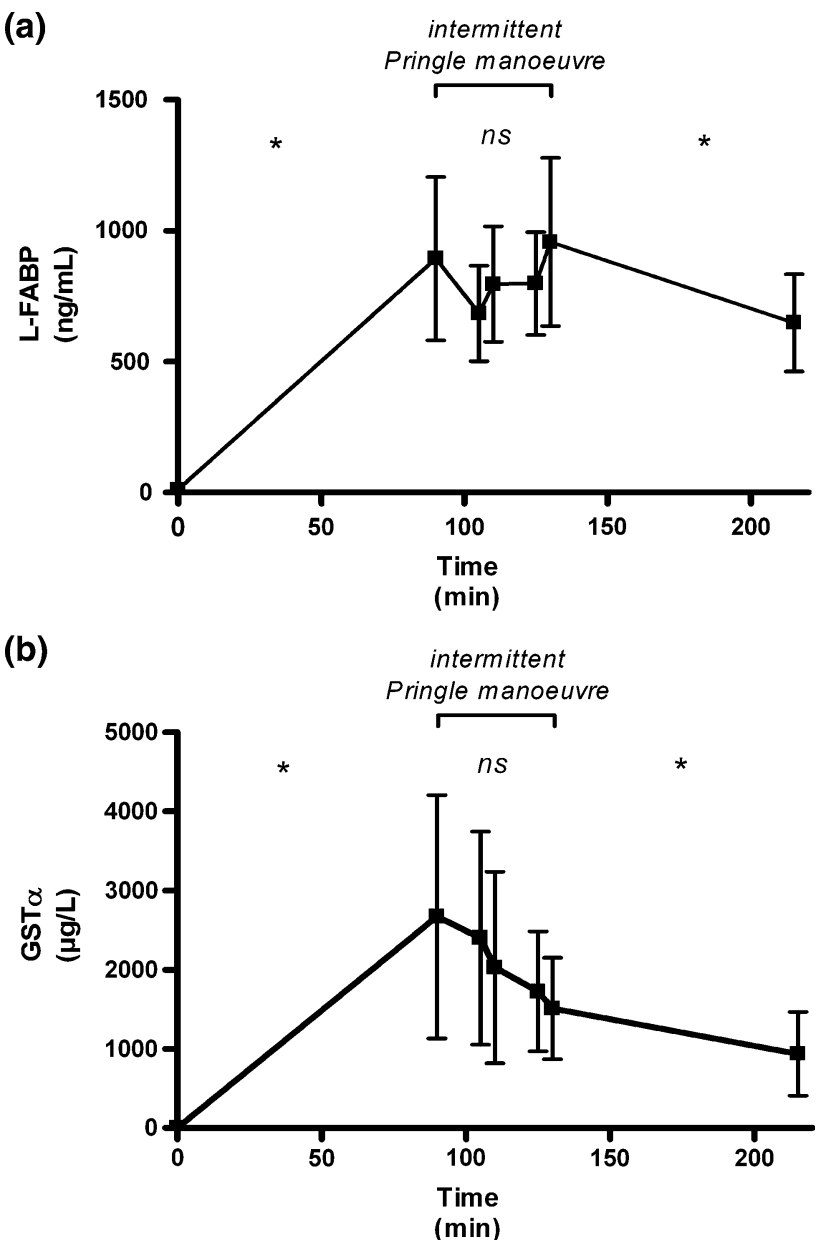

(c)

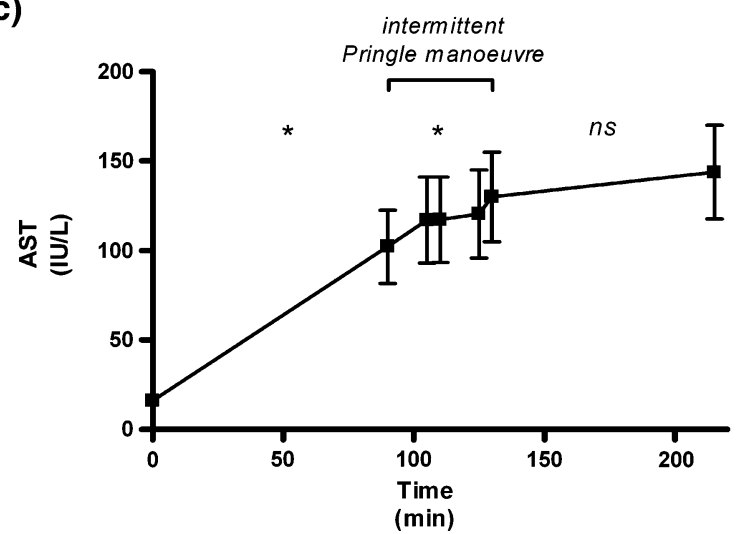

Fig. 1 Mean (SEM) arterial L-FABP (a), GST $\alpha$ (b), and AST (c) levels in patients undergoing liver surgery with intermittent Pringle maneuver $(n=9)$. Plasma levels of all liver injury markers increased early during surgery, during liver manipulation $(p<0.05)$. Intermittent Pringle maneuver $(2 \times 15 \mathrm{~min}$ ischemia and $5 \mathrm{~min}$ reperfusion $)$ did not cause significant changes in arterial L-FABP or GST $\alpha$ levels, whereas AST gradually kept increasing. L-FABP and GST $\alpha$ levels decreased immediately postoperatively ( $p=0.03$ and $p=0.004$, respectively). ${ }^{*} p$ $<0.05, \mathrm{~ns}=$ not significant; $\mathrm{I}=$ ischemia, $\mathrm{R}=$ reperfusion

significantly aggravated the hepatocellular injury caused by early perioperative processes. Immediately postoperatively, systemic L-FABP levels were found to be decreasing. 


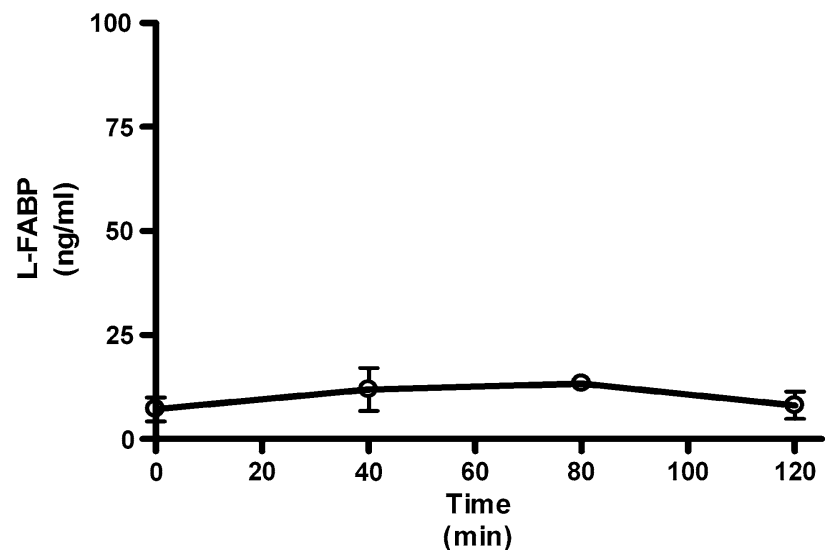

Fig. 2 Mean (SEM) plasma L-FABP levels in four patients undergoing lower abdominal surgery without manipulation of the liver. No changes in L-FABP concentration were observed

Systemic plasma levels of GST $\alpha$ (Fig. 1b) and AST (Fig. 1c) also increased significantly before liver transection and before ischemia-reperfusion.

\section{Effects of lower abdominal surgery on liver injury}

In patients undergoing lower abdominal surgery, the mean LFABP concentration remained below $15 \mathrm{ng} / \mathrm{ml}$, underlining the effect of direct manipulation of the liver on the elevation of L-FABP plasma levels during liver surgery (Fig. 2).

\section{Organ-specific FABP release}

Since L-FABP is expressed in both the liver and the intestines, we performed an organ balance analysis to reveal the origin of circulating L-FABP. The data show that L-FABP was specifically released from the liver and not from the intestines. In addition, organ balance analysis showed an active renal uptake of L-FABP from circulation (Fig. 3).

\section{Renal clearance of FABPs}

The kidneys efficiently removed L-FABP from circulation. Renal clearance of L-FABP correlated directly with their respective arterial concentrations (Fig. 4), resulting in a fractional extraction rate (arteriovenous gradient/arterial concentration $\times 100 \%$ ) [11] of approximately $30 \%$. Assuming that renal blood flow per minute approximates $22 \%$ of the total blood volume [12], renal plasma L-FABP clearance can be calculated to equal $6.6 \% / \mathrm{min}(30 \% \times$ $22 \% / \mathrm{min}$ ), resulting in a plasma half-life of about $11 \mathrm{~min}$.

Interleukin-6 levels during liver surgery with intermittent Pringle maneuver

The inflammatory response to liver surgery was investigated using IL-6 as a parameter. Arterial IL-6 levels

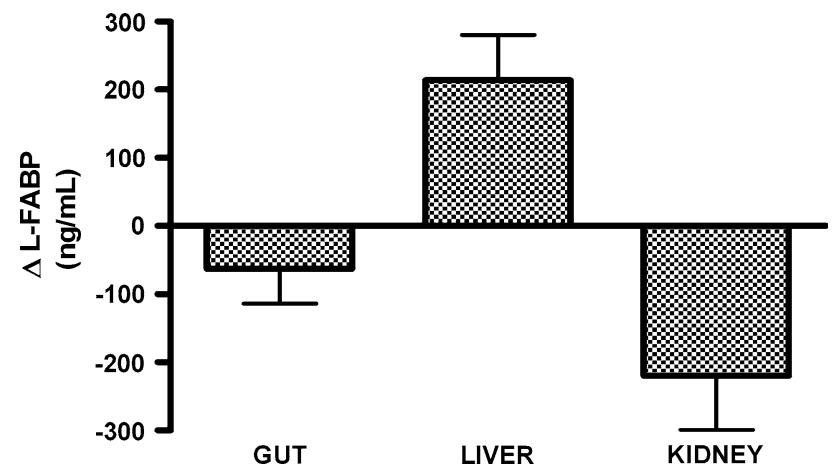

Fig. 3 Mean (SEM) arteriovenous concentration gradients of LFABP across the intestines (portal vein minus artery), the liver (hepatic vein minus portal vein), and the kidney in patients undergoing liver resection $(n=10)$. L-FABP was specifically released from the liver ( $p<0.0001$ vs. zero) and removed from circulation by the kidneys ( $p<0.0001$ vs. zero)

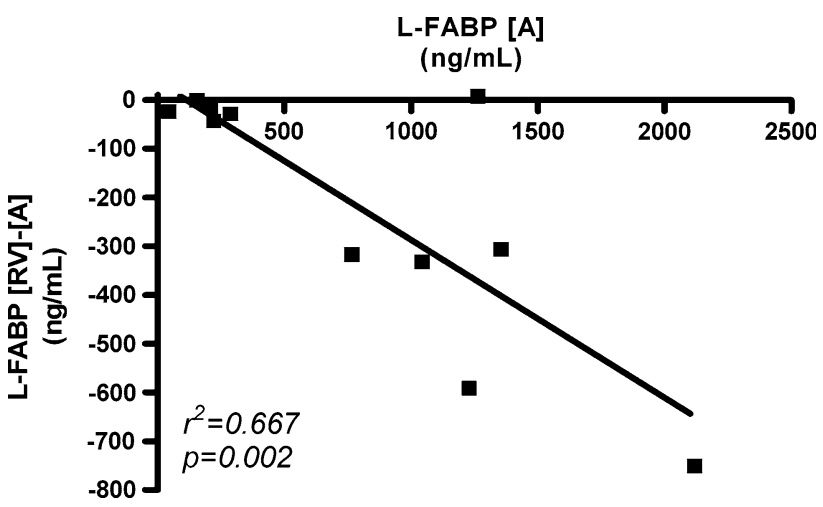

Fig. 4 Fractional renal clearance of L-FABP in patients undergoing liver resection $(n=10)$. From the fractional renal extraction of FABPs (approximately 30\%) and renal blood flow (22\% of total blood volume per minute) [12], a plasma half-life of FABPs of 11 min can be calculated. $[\mathrm{A}]=$ arterial concentration, $[\mathrm{RV}]=$ renal venous concentration

remained unaltered during manipulation of the liver, when L-FABP, AST, and GST $\alpha$ levels were increasing. In contrast, a significant increase of systemic IL-6 plasma levels was found after $15 \mathrm{~min}$ of hepatic inflow occlusion (Fig. 5). This increase was progressive throughout the remainder of the study period.

\section{Discussion}

The present study was designed to determine the cause of early hepatocellular injury during liver resection. Previous publications already showed elevated plasma levels of markers of liver damage prior to liver transection and hepatic inflow occlusion $[3,6]$. These observations suggested that mechanisms other than ischemia-reperfusion injury could contribute to peri- and postoperative cell death and 


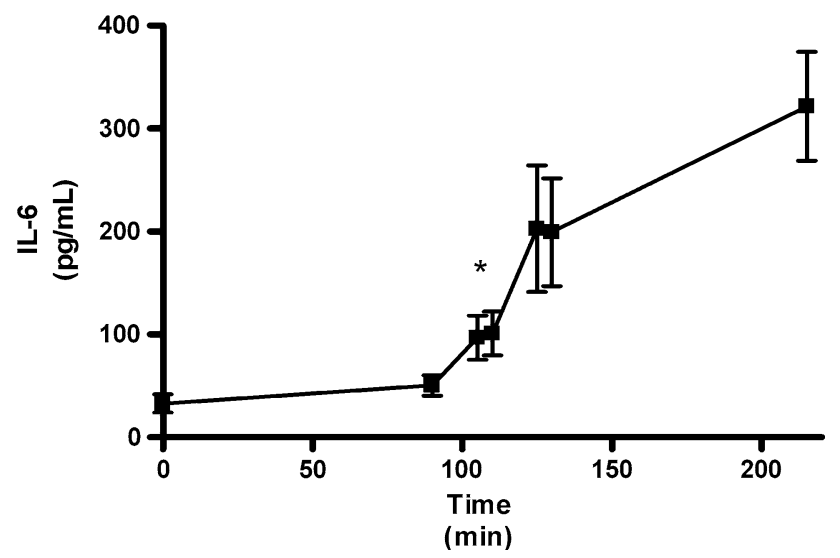

Fig. 5 Mean (SEM) arterial IL-6 concentration before, during, and after liver resection with intermittent Pringle maneuver $(n=9)$. * $=$ first timepoint where $p<0.05$ vs. baseline; $\mathrm{I}=$ ischemia, $\mathrm{R}=$ reperfusion

organ dysfunction. We showed that direct manipulation of the liver during surgery is a leading cause of hepatocellular injury.

Arterial L-FABP and GST $\alpha$ plasma levels increased following the start of the operation and reached a plateau before liver transection and ischemia-reperfusion. Surprisingly, we found no additional effect of organ transection or intermittent Pringle maneuver on these increased LFABP and GST $\alpha$ plasma levels. The apparent resistance of the liver to an ischemic insult however, is in line with previous data by Figueras et al. [13] who showed no effect of the extent of inflow occlusion in patients with a normal liver, although in their study livers of patients with cirrhosis appeared to be more vulnerable to hepatic inflow occlusion. In addition, other authors who showed a progressive release of GST $\alpha$ following a Pringle maneuver applied prolonged and continuous inflow occlusion [6], which is known to aggravate hepatocellular injury compared to the intermittent Pringle maneuver [14].

Previous authors who observed an early increase in plasma concentrations of transaminases and GST $\alpha$ have ascribed this phenomenon to the hepatotoxic effects of anesthesia [4], systemic inflammation after intestinal manipulation [5], and the effects of manipulation of the liver itself during perihepatic dissection and mobilization [6]. We were able to rule out the first two factors as potential causes of early hepatocyte injury since a similar increase of L-FABP plasma concentrations did not occur in patients undergoing lower intestinal surgery. These patients were anesthetized in a similar manner as the patients undergoing liver resection and underwent extensive intestinal manipulation.

By elimination of other potential causes, it thus must be concluded that increasing L-FABP levels occurs during and due to liver manipulation in patients undergoing liver resection. By measuring concentration gradients across the intestines and the liver, we were able to show that the increased L-FABP levels during liver manipulation are exclusively due to L-FABP release from the liver. Some intestinal L-FABP release could have been expected when L-FABP, which is also expressed by the intestines, would have been released from injured enterocytes, but the absence of intestinal L-FABP release clearly indicates that organ manipulation during liver resection results in hepatocellular injury without causing intestinal injury.

Finally, we were able to rule out systemic inflammation as a leading cause of hepatocellular injury since L-FABP peak levels were reached already before the onset of the inflammatory response. Arterial IL-6 plasma levels increased between 90 and 125 min after laparotomy, which approximates the established time lag between an inflammatory stimulus and IL-6 release [15]. This suggests that the inflammatory response is triggered by an early event during the operation.

According to the "danger model" hypothesis of Matzinger [16], cell injury leads to the release of immunostimulatory proteins or nucleotides, so-called danger signals, that activate the immune system and induce systemic inflammation [17]. In line with this theory, we consider that early hepatocyte damage due to liver manipulation could give rise to the release of such "danger signals" and contribute to systemic inflammation. Consequently, liver manipulation-induced hepatocyte injury may be a trigger for the inflammatory response to surgery. Mechanistic proof for this theory, however, is difficult to obtain in vivo.

Both L-FABP and GST $\alpha$ decreased immediately following surgery, in contrast to the more classic marker of hepatocyte injury, AST. The ongoing increase of plasma levels of such markers following liver surgery has been regarded as a sign of ongoing hepatocellular injury and impending liver failure [18]. Our study shows that the late postoperative peak of AST is more likely to be a reflection of slow leakage than of ongoing injury, since the leakage of small-molecular proteins L-FABP and GST $\alpha$ decreased within $90 \mathrm{~min}$. As a consequence, L-FABP and GST $\alpha$ are probably more sensitive for detecting ongoing hepatocyte injury and impending liver failure than AST. To prove this assumption, however, a large prospective study is needed. The rapid decline of L-FABP and GST $\alpha$ is a result of their rapid renal clearance. Arterial renal venous concentration gradients showed that the kidneys remove approximately $30 \%$ of L-FABP in a single pass, leading to a calculated LFABP half-life of $11 \mathrm{~min}$.

We did not explore potential mechanisms of liver manipulation-induced cell injury. Earlier studies showed that liver mobilization and assessment of resectability significantly reduced hepatic venous oxygen saturation [19, 
20]. This could also be a cause of hepatocyte damage in our case, although we did not measure hepatic oxygen saturation. It is not clear whether hepatic oxygen saturation decreased due to physical obstruction of the blood stream or whether it decreased secondary to other processes [21, 22]. Alternatively, cell damage may occur as a direct consequence of mechanical impact [23].

In summary, it was previously believed that vascular occlusion and parenchymal transection were the major reasons for hepatocyte injury during liver surgery. This study demonstrates that liver manipulation is a leading cause of hepatocyte injury during liver surgery. A potential causal relation between liver manipulation and systemic inflammation remains to be established. However, since the inflammatory cascade is apparently initiated early during major abdominal surgery, interventions aimed at reducing postoperative inflammation and related complications should be started early during surgery or beforehand.

Acknowledgments The authors thank M. Hadfoune and A.A. van Bijnen for their skillful help in our laboratory. This study was supported by grants from The Netherlands Organization for Health Research and Development to MCGvdP (920-03-317 AGIKO) and CHCD (907-00-033 Clinical Fellowship).

\section{References}

1. Wigmore SJ, Redhead DN, Yan XJ, et al. (2001) Virtual hepatic resection using three-dimensional reconstruction of helical computed tomography angioportograms. Ann Surg 233:221-226

2. Patel A, van de Poll MC, Greve JW, et al. (2004) Early stress protein gene expression in a human model of ischemic preconditioning. Transplantation 78:1479-1487

3. Boschetto A, Dondero F, Tonini V, et al. (2005) Intra-operative liver injury is not only related to vascular clamping [abstract]. HPB 7:P25

4. Clarke RS, Doggart JR, Lavery T (1976) Changes in liver function after different types of surgery. Br J Anaesth 48:119-128

5. Hiki N, Shimizu N, Yamaguchi H, et al. (2006) Manipulation of the small intestine as a cause of the increased inflammatory response after open compared with laparoscopic surgery. Br J Surg 93:195-204

6. Chouker A, Martignoni A, Schauer RJ, et al. (2005) a-Gluthathione s-transferase as an early marker of hepatic ischemia/reperfusion injury after liver resection. World J Surg 29:528-534

7. Dejong CHC, Garden OJ (2003) Neoplasms of the liver. In: Majid AA, Kingsnorth A (eds) Advanced surgical practice. London, Greenwich Medical Media, pp 146-156
8. Mulder TP, Peters WH, Court DA, et al. (1996) Sandwich ELISA for glutathione S-transferase alpha 1-1: plasma concentrations in controls and in patients with gastrointestinal disorders. Clin Chem 42:416-419

9. Steegers EA, Mulder TP, Bisseling JG, et al. (1995) Glutathione S-transferase alpha as marker for hepatocellular damage in preeclampsia and HELLP syndrome. Lancet 345:1571-1572

10. Motulsky HJ (2003) Comparing a mean or median to a theoretical value. Prism 4 Statistics Guide: Statistical analyses for laboratory and clinical researchers. GraphPad Software Inc., San Diego, CA, pp 35-39

11. van de Poll MC, Siroen MP, van Leeuwen PA, et al. (2007) Interorgan amino acid exchange in humans: consequences for arginine and citrulline metabolism. Am J Clin Nutr 85:167-172

12. Riddez L, Hahn RG, Brismar B, et al. (1997) Central and regional hemodynamics during acute hypovolemia and volume substitution in volunteers. Crit Care Med 25:635-640

13. Figueras J, Llado L, Ruiz D, et al. (2005) Complete versus selective portal triad clamping for minor liver resections: a prospective randomized trial. Ann Surg 241:582-590

14. Belghiti J, Noun R, Malafosse R, et al. (1999) Continuous versus intermittent portal triad clamping for liver resection: a controlled study. Ann Surg 229:369-375

15. Lin E, Calvano SE, Lowry SF (2000) Inflammatory cytokines and cell response in surgery. Surgery 127:117-126

16. Matzinger $P$ (2002) The danger model: a renewed sense of self. Science 296:301-305

17. Barrat FJ, Meeker T, Gregorio J, et al. (2005) Nucleic acids of mammalian origin can act as endogenous ligands for Toll-like receptors and may promote systemic lupus erythematosus. J Exp Med 202:1131-1139

18. Panis Y, McMullan DM, Emond JC (1997) Progressive necrosis after hepatectomy and the pathophysiology of liver failure after massive resection. Surgery 121:142-149

19. Kretzschmar M, Kruger A, Schirrmeister W (2003) Hepatic ischemia-reperfusion syndrome after partial liver resection (LR): hepatic venous oxygen saturation, enzyme pattern, reduced and oxidized glutathione, procalcitonin and interleukin-6. Exp Toxicol Pathol 54:423-431

20. Kainuma M, Fujiwara Y, Kimura N, et al. (1991) Monitoring hepatic venous hemoglobin oxygen saturation in patients undergoing liver surgery. Anesthesiology 74:49-52

21. Schemmer P, Enomoto N, Bradford BU, et al. (2001) Activated Kupffer cells cause a hypermetabolic state after gentle in situ manipulation of liver in rats. Am J Physiol Gastrointest Liver Physiol 280:G1076-1082

22. Schemmer P, Enomoto N, Bradford BU, et al. (2001) Autonomic nervous system and gut-derived endotoxin: involvement in activation of Kupffer cells after in situ organ manipulation. World J Surg 25:399-406

23. Lazarowski ER, Homolya L, Boucher RC, et al. (1997) Direct demonstration of mechanically induced release of cellular UTP and its implication for uridine nucleotide receptor activation. J Biol Chem 272:24348-24354 